\title{
Lacrimogenic Effects of Cyclosporine given per Os in Dogs with Keratoconjunctivitis Sicca
}

\author{
David L Williams* MA MEd Vet MD PhD DECAWBM CertVOphthal FRCVS \\ Department of Veterinary Medicine, University of Cambridge, Madingley Road, Cambridge CB3 OES, UK \\ *Corresponding Author: Dr Williams, Department of Veterinary Medicine, University of Cambridge, \\ Madingley Road, Cambridge CB3 OES, UK, Email: dlw33@ cam.ac.uk
}

\begin{abstract}
The effects of oral cyclosporine on tear production in dogs with keratoconjunctivitis sicca (KCS) were evaluated in $20 \mathrm{KCS}$-affected dogs. Dogs were given a full ophthalmic examination with bilateral Schirmer tear test (STT) readings taken together with evaluation of blepharospasm, conjunctival hyperaemia, ocular discharge, corneal opacification, vascularisation and pigmentation scored between 0 (none) and 4 (severe), these summated to give a total clinical score. Dogs were treated with topical carbomer gel (Viscotears: Novartis Ophthalmics) three times daily and oral cyclosporine (Atopica: Novartis) $5 \mathrm{mg} / \mathrm{kg}$ once daily for the first 42 days, then every other day for the next 28 days then twice weekly to the end of the study. Dogs were removed from the trial if ocular surface pathology did not improve or deteriorated or if gastrointestinal side effects were marked. STT rose from $5.7 \pm 2.3 \mathrm{~mm} / \mathrm{min}$ to $10.7 \pm 5.9 \mathrm{~mm} / \mathrm{min}(p<0.0001)$ with daily cyclosporine administration falling to $8.7 \pm 6.0 \mathrm{~mm} / \mathrm{min}(p=0.017)$ on twice weekly administration. Total clinical score fell from 8.0 13.3 to $4.6 \pm 3.0(p=0.0019)$ with daily cyclosporine administration rising to $5.6 \pm 3.9$ $(p=0.0014)$ on twice weekly administration. Two dogs were withdrawn for reasons of inefficacy during the study, one with poor owner compliance, one because of drug side effects of vomiting and diarrhoea while two dogs died of unrelated causes during the study. These results show that cyclosporine given per os has a lacrimogenic action which may be useful in dogs where topical application is difficult.
\end{abstract}

\section{INTRODUCTION}

It was nearly twenty years ago that topical $2 \%$ cyclosporine in corn oil was first reported as a lacrimogenic agent in canine kerato conjunctivitis sicca (KCS) (Kaswan et al., 1989). Since then other workers have repeated this initial work, using the immunomodulatory drug at lower concentrations (Olivero et al., 1991) and in different vehicles (Sansom et al., 1995) as well as investigating other drugs such as tacrolimus (Berdoulay et al., 2005, Radziejewski and Balicki 2016) and pimecrolimus (Nell et al., 2005) with similar immunomodulatory mechanisms. Twenty years ago the only regularly used medical treatments for canine KCS were regular tear replacement with topical medications such as carboxymethylcellulose drops, carbomer gel or hyaluronic acid (Williams et al., 2012 Williams and Mann 2014). These did not seek to target the cause of the condition, most notably an immune-mediated destruction of lacrimal tissue (Kaswan et al., 1984, Williams 2008, Williams and Tighe 2018) but merely ameliorated resulting ocular surface pathology. Topical pilocarpine has beneficial effects in increasing tear production in some animals (Slatter and Severin 1995) but its narrow therapeutic window meant that successful treatment was often accompanied by undesirable side effects. The surgical option of parotid duct transposition, widely used before the advent of topical cyclosporine, could be highly effective (Glen and Dawson 1971), and is still used today, mostly in cyclosporine-resistant neurological cases but in a sizeable minority gland atrophy or duct obstruction leads to failure of the operation long-term and in others over-production of saliva results in epiphora and facial scalding (Termote 2003)..

The introduction of topical cyclosporine at $2 \%$ in corn oil led to a licensed product, Optimmune (Shering-Plough, New Jersey), containing cyclosporine at $0.2 \%$ in an ointment base, vastly improving the commercially available therapeutic options for canine KCS. Development of a similar drug for human patients took a considerable time but Resistas is more and more widely used as a topical lacrimogen for people with dry eye (Perry et al., 2008). Yet Dr Kaswan's original interest in using the drug stemmed from the experience of 
a friend who, treated with cyclosporine per os after a renal transplant, experienced excess lacrimation (Kaswan pers comm 2015). This led Dr Kaswan to investigate the value of topical cyclosporine as a lacrimogen with the remarkable success that has led to a revolution in therapy of dry eye in both dogs (Williams 1997) and in man eye (Perry et al., 2008). Since Kaswan's first report, as we have noted above, numerous subsequent reports have documented the use of the drug topically. None, however, has investigated the lacrimogenic potential of cyclosporine delivered per os. The drug has been widely used systemically as a veterinary product for diseases from atopic dermatitis (Olivry et L., 2002) to anal furunculosis (Hardie et al., 2005) and its safety record is widely known (Stefan et al., 2005).

In a number of canine KCS patients owners find regular topical administration difficult and the option of giving cyclosporine per os would be welcomed, were it to have the same lacrimogenic properties it does by topical application. Here we aimed to investigate the lacrimogenic effects of cyclosporine delivered per os in a controlled but not masked study carefully conducted under the auspices of good clinical practice (Salzberg and Muller 2003).

\section{Materials ANd Methods}

Twenty dogs from the Queen's Veterinary School Hospital, University of Cambridge, UK and a number of first opinion veterinary clinics for which DW provides an ambulatory ophthalmological service were included in the study out of 76 dogs seen with KCS in the study period. Inclusion criteria included the presence of bilateral keratoconjunctivitis sicca with a Schirmer tear test of $1-10 \mathrm{~mm} / \mathrm{min}$, this higher level being the recognised cut-off for the diagnosis of the disease (Hartley et al., 2006). Dogs with absolute dry eye and an STT of zero or those which has previously been treated with topical cyclosporine were excluded from the trial, as were those with corneal ulceration and animals with systemic diseases, as detailed in table 1. Withdrawal criteria included worsening of tear production or ocular surface health or uncontrollable side effects such as emesis or diarrhoea.

The aim of the study was to evaluate both tear production, as measured by the Schirmer tear test (STT) (Gelatt et al., 1975) and ocular surface health as determined by a battery of scores of ocular surface pathology, namely blepharospasm, conjunctival hyperaemia, ocular discharge, corneal opacification, vascularisation and pigmentation before and during per os cyclosporine treatment. Initially given at the standard dose of $5 \mathrm{mg} / \mathrm{kg}$ given once daily, the drug was gradually reduced in a tapering regime, being given once daily for the first 42 days, then every other day for the next 28 days then twice weekly to the end of the study. The carbomer gel tear replacement Viscotears (Allergan New York) was given three times daily in each eye but not on the day of ophthalmic examination. Changes in tear production and ocular surface health were documented on a regular basis with examinations at $0,14,28,42,70,84$ and 98 days involving direct and indirect ophthalmoscopy and slit lamp biomicroscopy together with the assessment of tear production with the Schimer tear test. Owner evaluation of changes in the animal's ocular health and behaviour were achieved by a standard questionnaire at each examination involving the amount of discharge, the degree of eye rubbing and squinting.

The statistical significance of changes STT values while on treatment were obtained using a Mann Whitney $U$ test given that the data were not normally distributed, with significance deemed to have been reached at $\mathrm{p}<0.05$.

\section{RESUltS}

The 20 dogs were of breeds as documented in table 2. 55\% were spayed females. The mean age of all dogs was 8.22 years with a range from 0.09 to 16.8 years. $80 \%$ of dogs had a body weight of $15 \mathrm{kgs}$ or less.

The mean STT for left and right eyes and the average of both is given in table 3 and shown graphically in figure 1. STT rose from an average of $5.7 \pm 2.3 \mathrm{~mm} / \mathrm{min}$ at the first examination before drug administration to a maximum of $10.7 \pm 5.9 \mathrm{~mm} / \mathrm{min}(\mathrm{p}<0.0001)$ at the fourth examination 42 days after the initiation of treatment with daily cyclosporine administration. STT fell to $9.5 \pm 5.7 \mathrm{~mm} / \mathrm{min}$ on cyclosporine treatment every other day, decreasing further to $8.7 \pm 6.0 \mathrm{~mm} / \mathrm{min}(\mathrm{p}=0.017)$ on twice weekly administration. 
Schirmer tear test (both eyes)

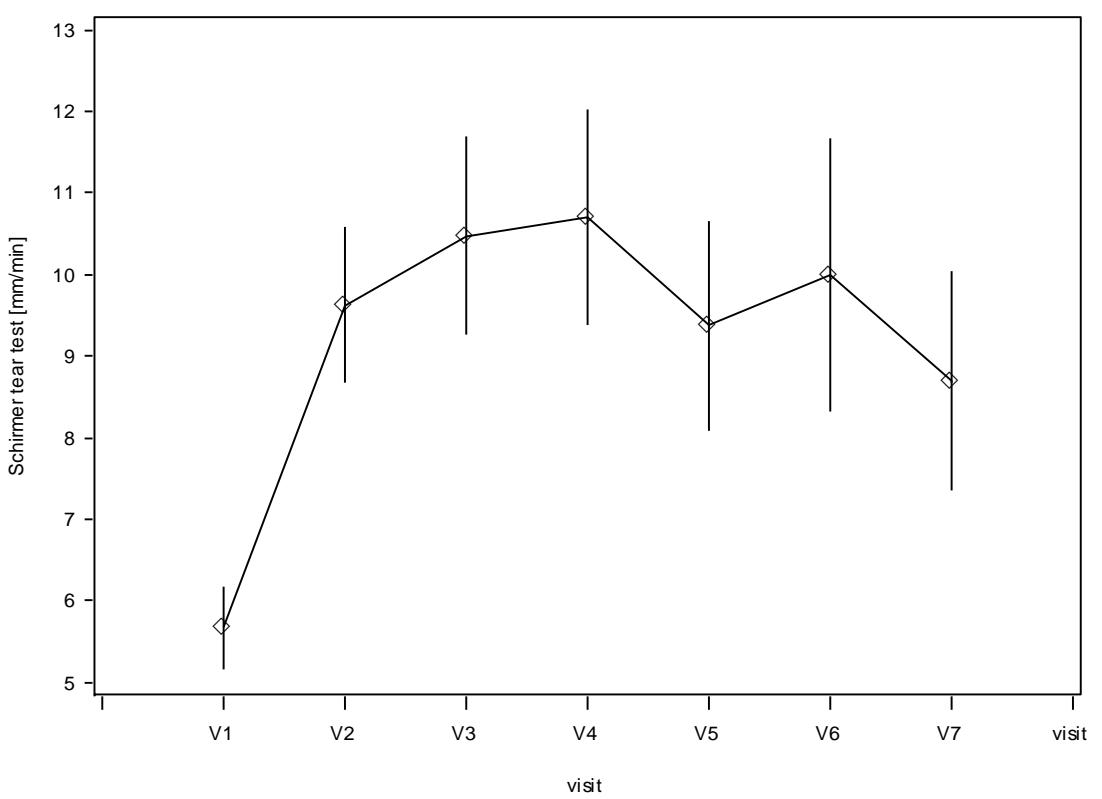

Figure 1. Schirmer tear test during Atopica medication once daily (Visit 1-4) and then in reduction to once every other day daily (visit 5-6) and then twice weekly (visit 6-7))

Mean values for measures of ocular surface health are shown graphically for blepharospasm in figure 2, conjunctival hyperaemia in figure 3 , corneal opacification and oedema in figure 4, corneal vascularisation in figure 5 , ocular discharge in figure 6 and corneal pigmentation in figure 7 . The total clinical score as a summation of the individual scores noted previously is shown in figure 8. Owner assessment of ocular discharge through the period of assessment is shown graphically in figure 9 , for eye rubbing in figure 10 and for squinting in figure 11.

\section{Blepharospasm (both eyes)}

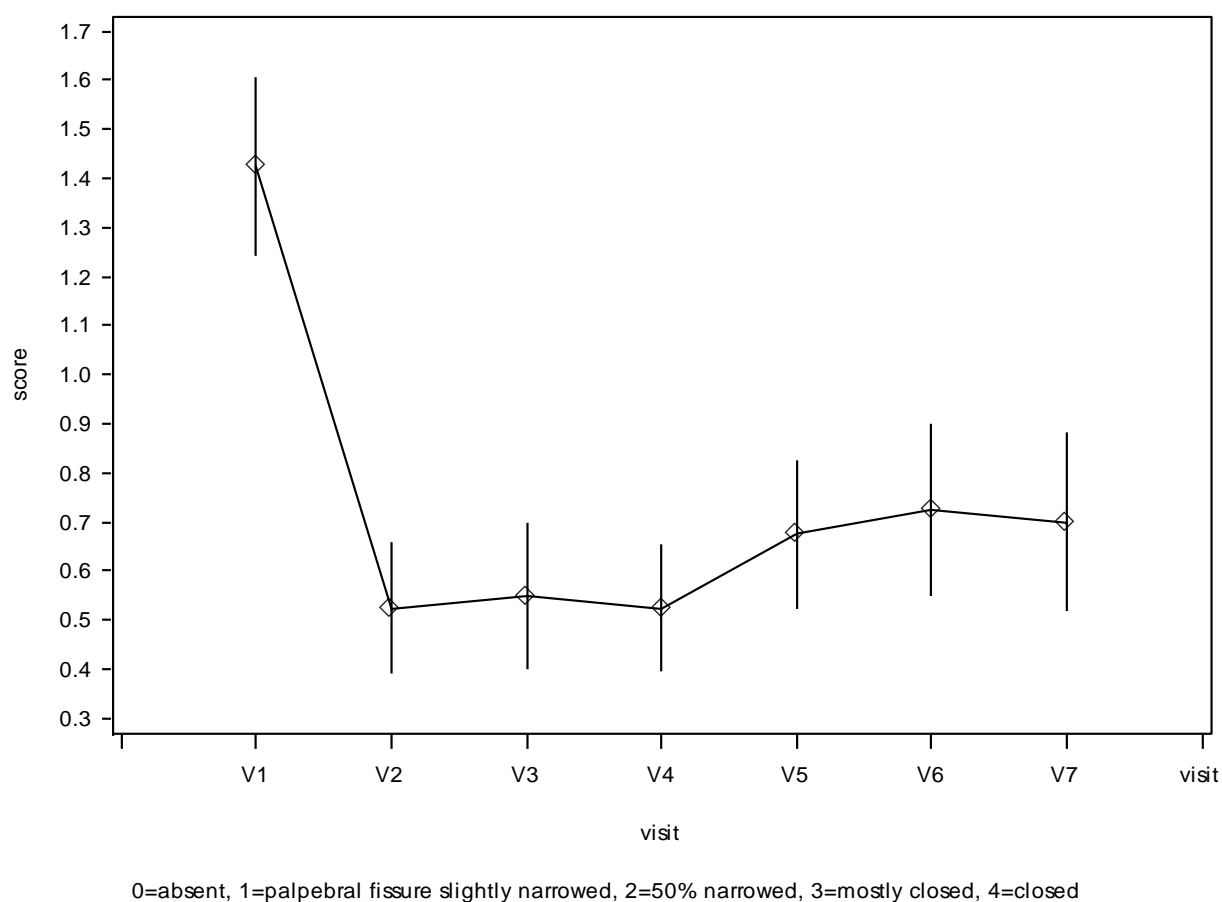

Figure 2. Blepharospasm score during Atopica medication once daily (Visit 1-4) and then in reduction to once every other day daily (visit 5-6) and then twice weekly (visit 6-7)) 
Conjunctival hyperaemia (both eyes)

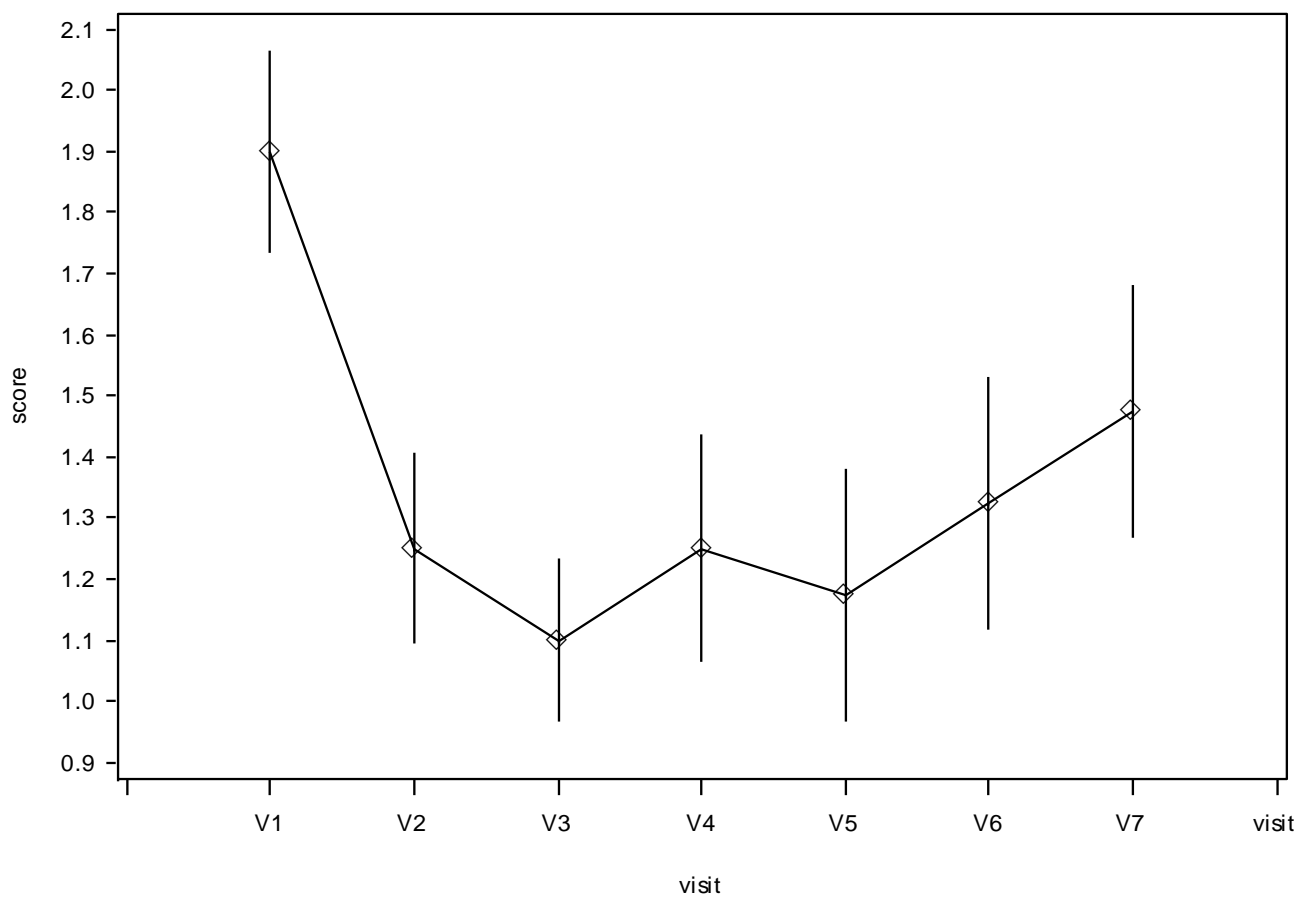

$0=$ absent, 1 =white sclera visible, $2=$ slightly obscured, $3=$ completely obscured, $4=$ deep conjunctival hyperaemia

Figure 3. Conjunctival hyperaemia score during Atopica medication once daily (Visit 1-4) and then in reduction to once every other day daily (visit 5-6) and then twice weekly (visit 6-7))

Corneal opacification / oedema (both eyes)

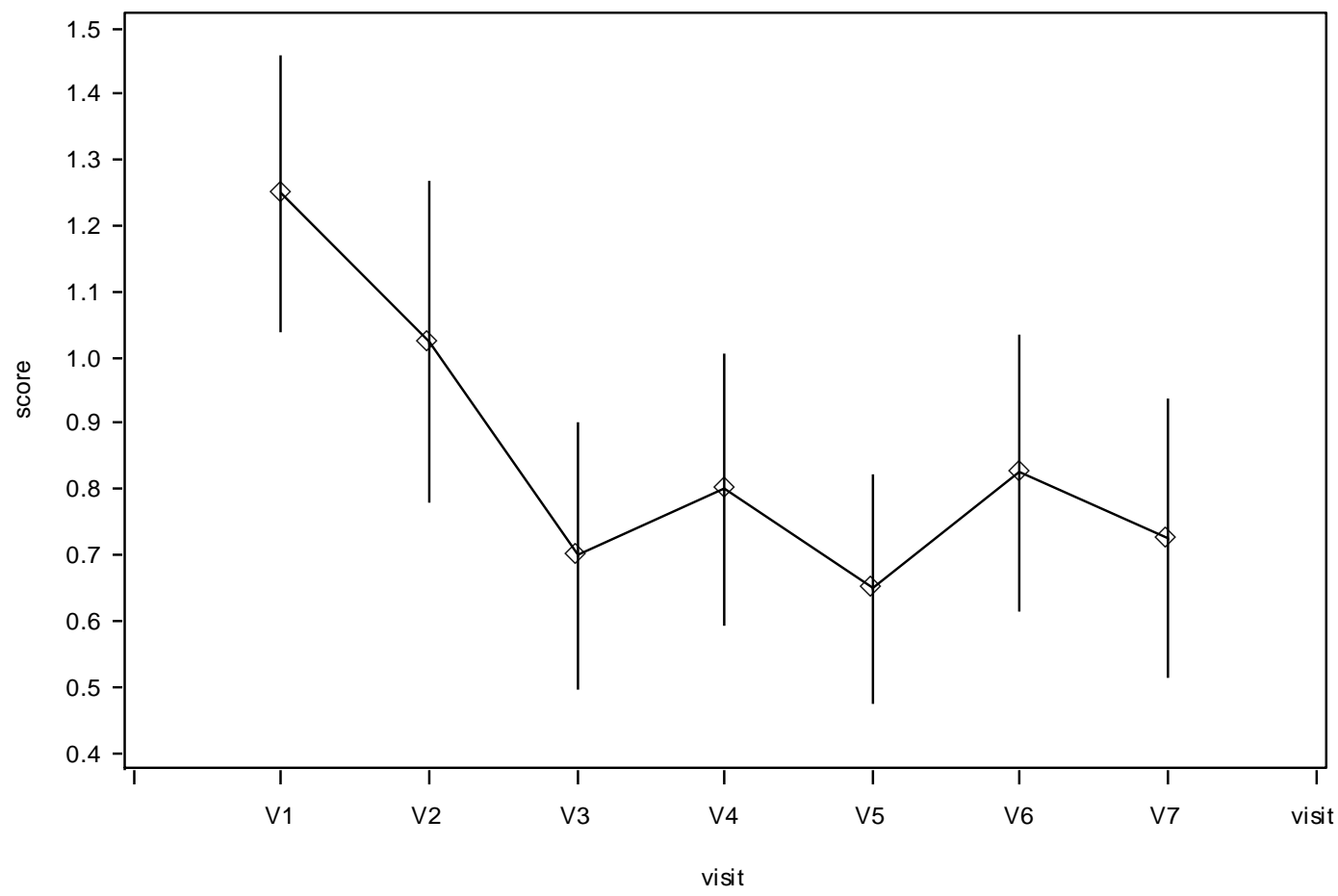

$0=$ clear cornea, $1=$ mild cornea haze, $2=$ moderate corneal opacity,

$3=$ severe corneal opacity, iris visible, $4=$ opaque cornea

Figure 4. Corneal opacification score during Atopica medication once daily (Visit 1-4) and then in reduction to once every other day daily (visit 5-6) and then twice weekly (visit 6-7)) 


\section{Corneal vascularization (both eyes)}

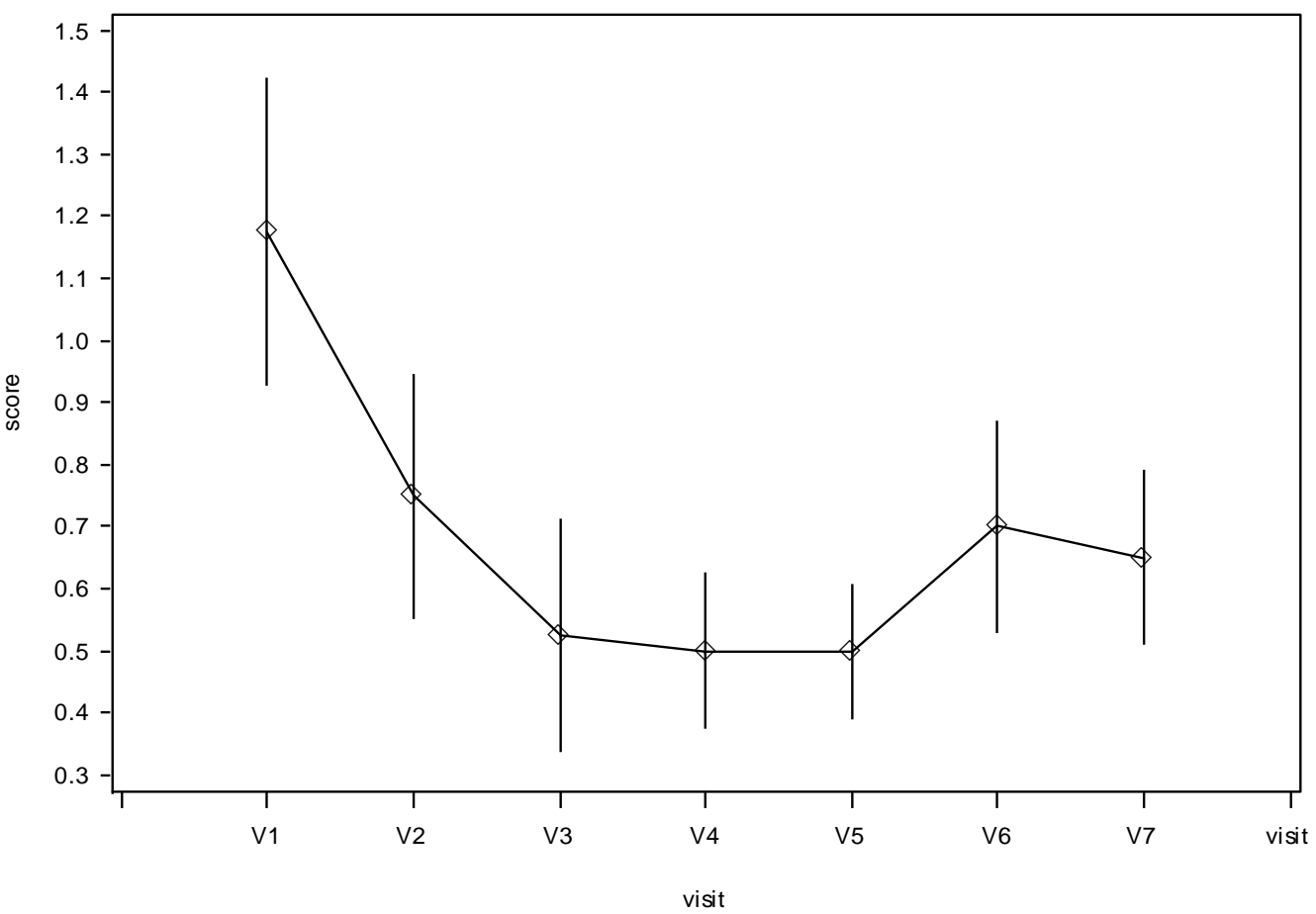

$0=$ absent, $1=$ less than $25 \%$ of cornea vascularized, $2=25$ to $50 \%, 3=50$ to $75 \%, 4=>75 \%$ of cornea vascularized

Figure 5. Corneal vascularization score during Atopica medication once daily (Visit 1-4) and then in reduction to once every other day daily (visit 5-6) and then twice weekly (visit 6-7))

\section{Amount of ocular discharge (both eyes)}

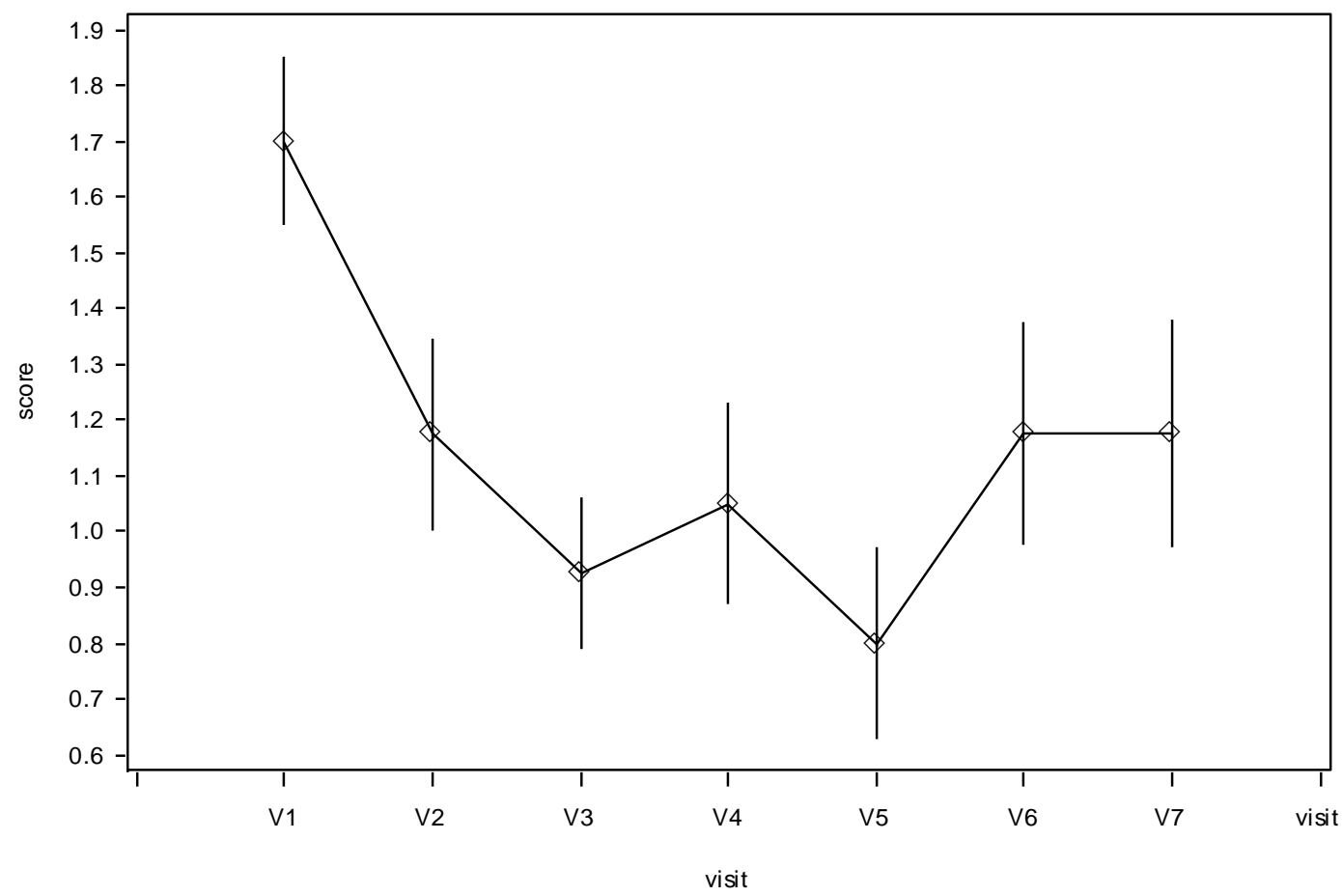

$0=$ no mucus present, $1=$ clear mucus, $2=$ thick mucus/crusting just at medial canthus, $3=$ across cornea, $4=0$ eyelids and face

Figure 6. Ocular discharge score during Atopica medication once daily (Visit 1-4) and then in reduction to once every other day daily (visit 5-6) and then twice weekly (visit 6-7)) 
Total KCS clinical score (both eyes)

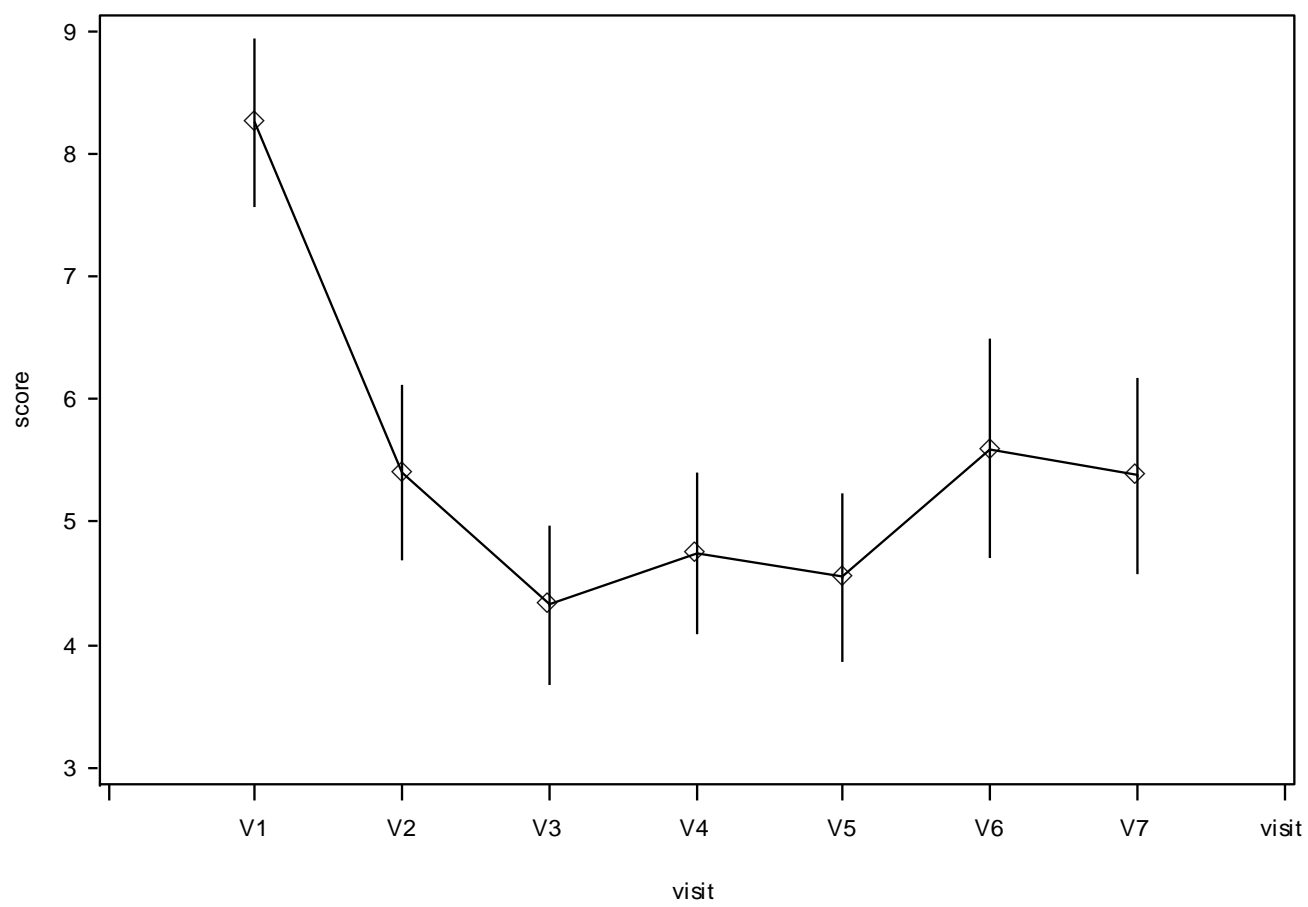

Scale is from $0=$ best to $24=$ worst

Figure 7. Overall clinical score during Atopica medication once daily (Visit 1-4) and then in reduction to once every other day daily (visit 5-6) and then twice weekly (visit 6-7))

\section{Amount of discharge (owner) (both eyes)}

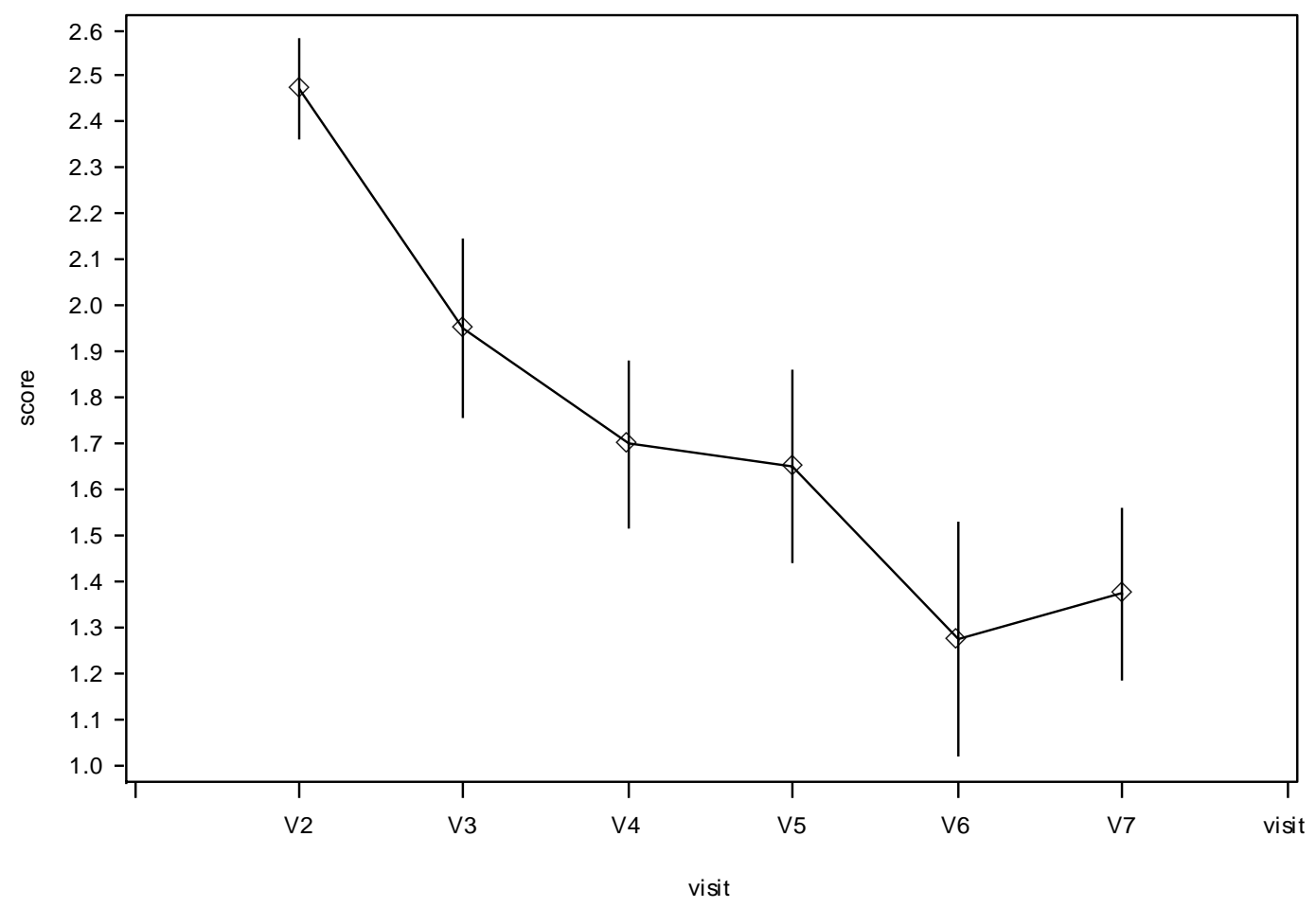

Scale is from $0=$ best to $3=$ worst

Figure 8. Owner assessment of ocular discharge during Atopica medication once daily (Visit 1-4) and then in reduction to once every other day daily (visit 5-6) and then twice weekly (visit 6-7)) 


\section{Rubbing (owner) (both eyes)}

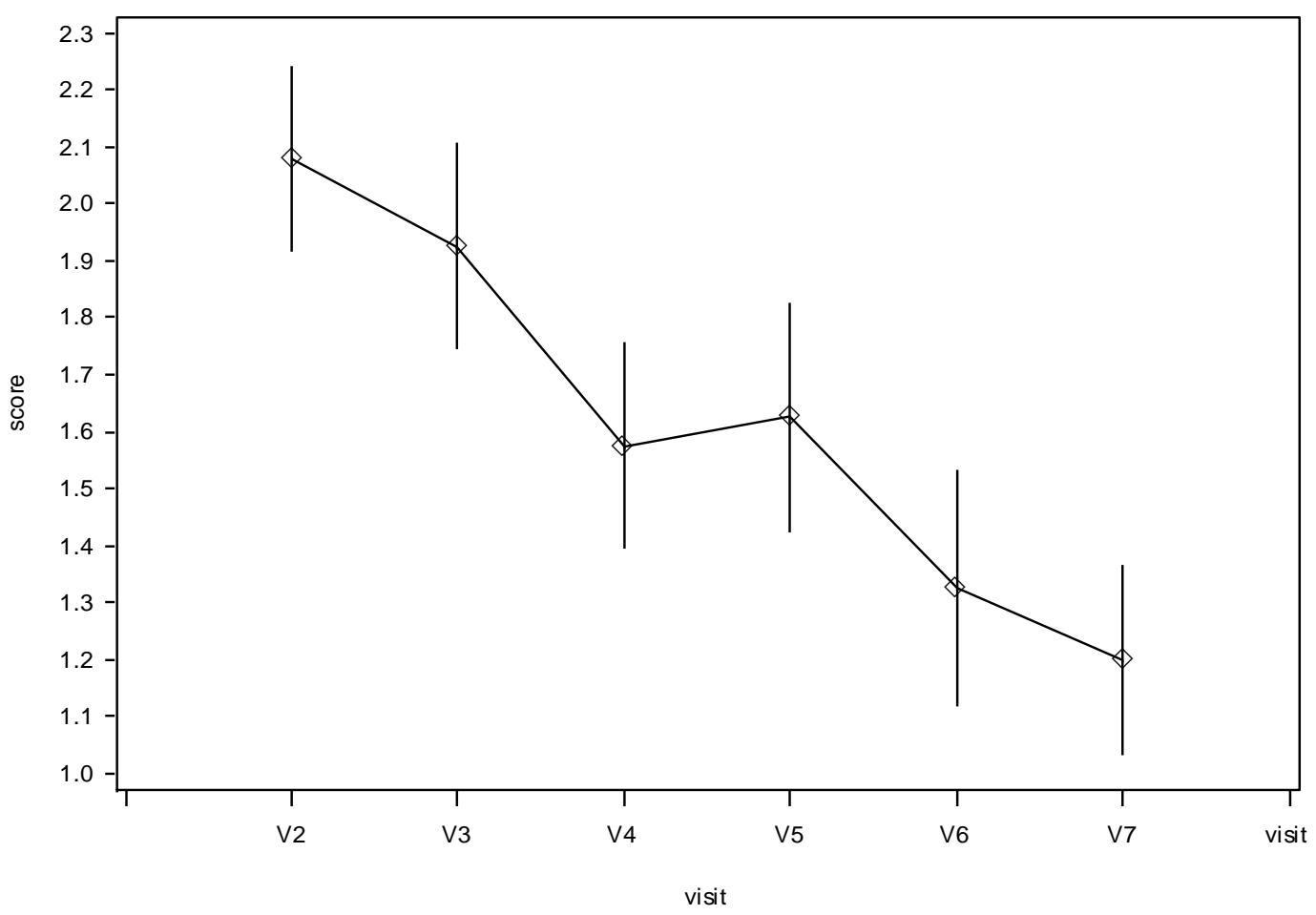

Scale is from $0=$ best to $3=$ worst

Figure 9. Ocular assessment of 'rubbing of eyes' during Atopica medication once daily (Visit 1-4) and then in reduction to once every other day daily (visit 5-6) and then twice weekly (visit 6-7))

Squinting (owner) (both eyes)

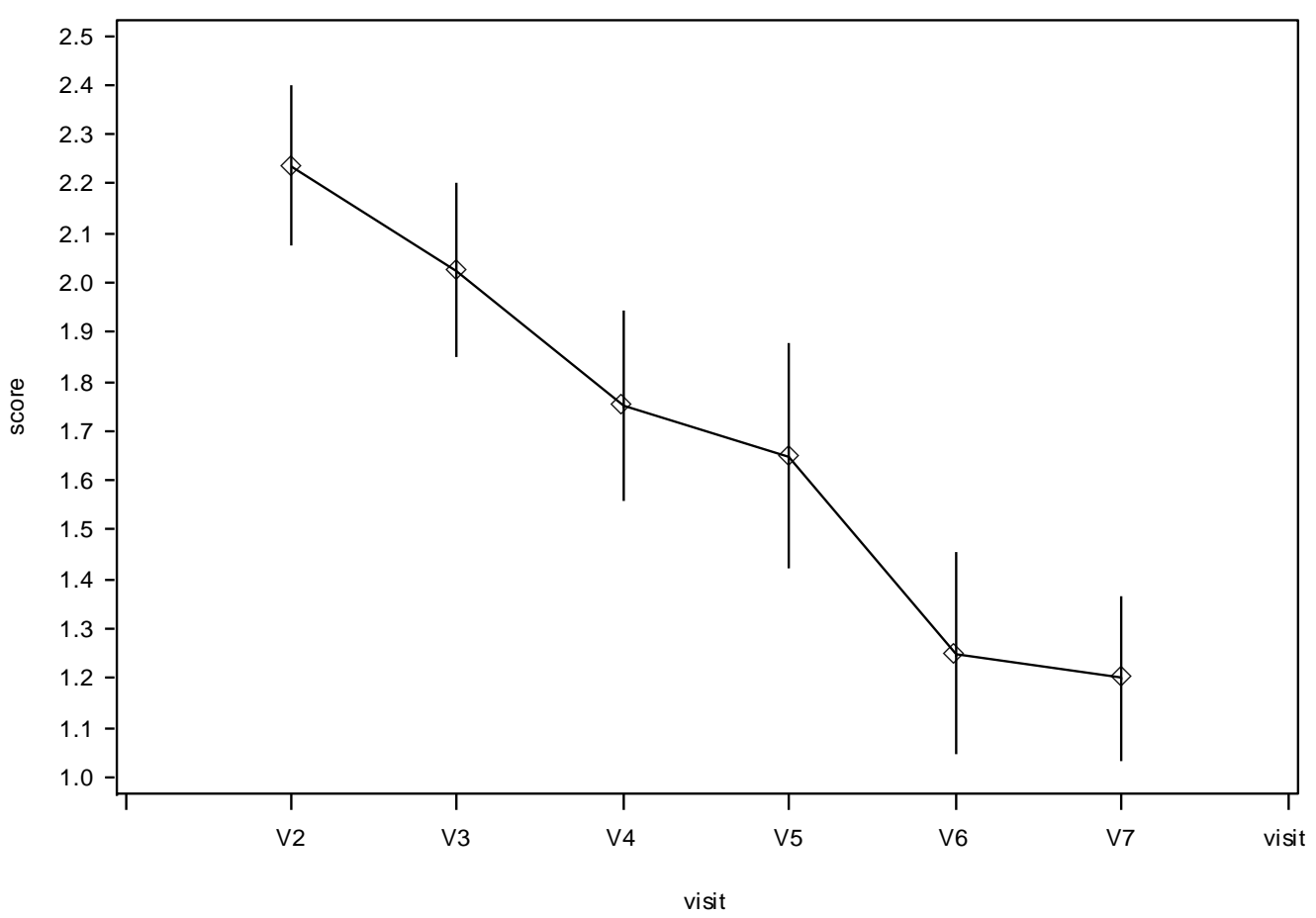

Scale is from $0=$ best to $3=$ worst

Figure 10. Ocular assessment of 'squinting of eyes' during Atopica medication once daily (Visit 1-4) and then in reduction to once every other day daily (visit 5-6) and then twice weekly (visit 6-7)) 


\section{Clinical improvement vs last visit}

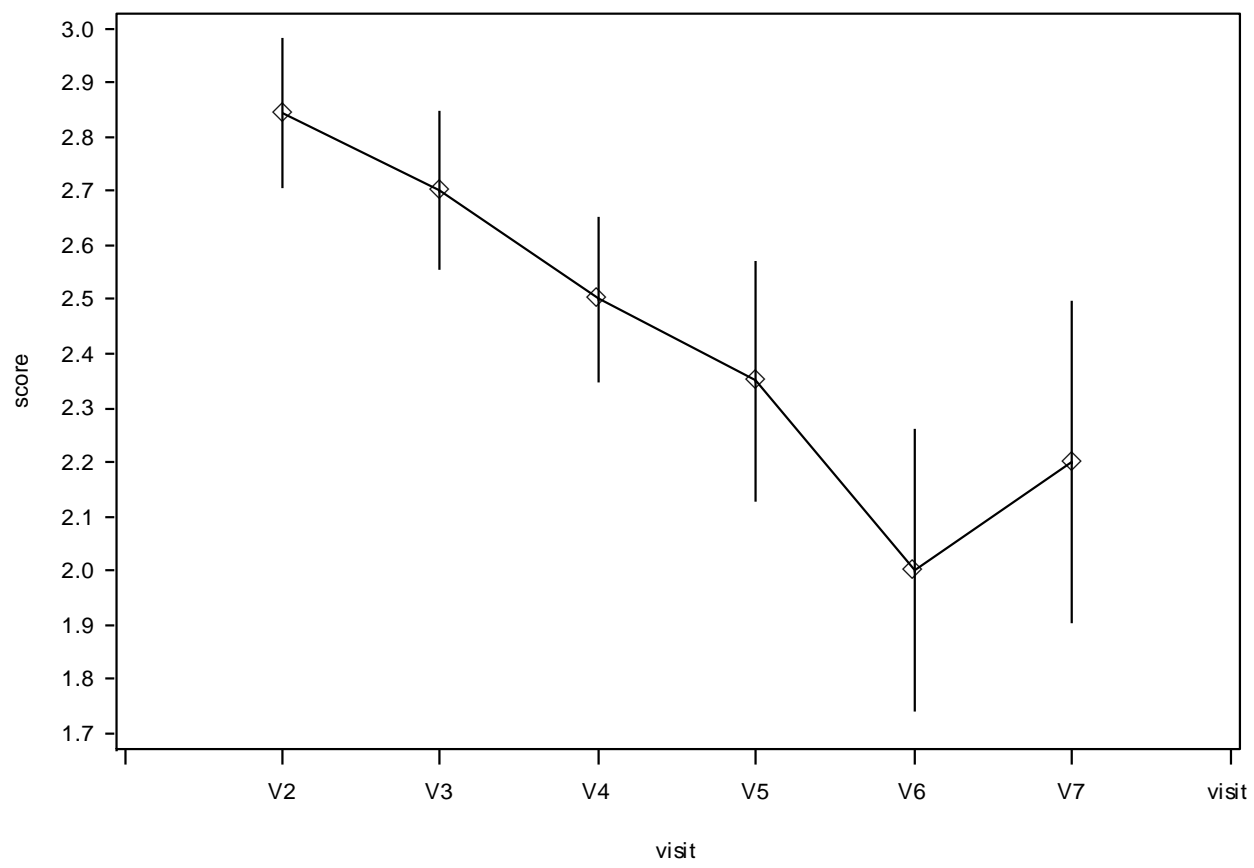

0 =bad, $1=$ poor, $2=$ =acceptable, $3=$ good, $4=$ excellent

Figure 11. Owner assessment of improvement in clinical signs since last visit during Atopica medication once daily (Visit 1-4) and then in reduction to once every other day daily (visit 5-6) and then twice weekly (visit 6-7))

As might be anticipated from the improvement in tear production on daily treatment and the subsequent fall as administration frequency is reduced, the clinical scores were lower on daily treatment, rising somewhat on every other day and twice weekly treatment. Importantly, even on the reduced frequency of twice weekly, the scores were improved from those seen at the initiation of treatment and for every examination apart form the last after four weeks of twice weekly treatment, the condition was determined to be improved form that of the previous visit.

Six dogs failed to complete the course of treatment. Two were withdrawn because of lack of drug efficacy after reduction in administration frequency to twice weekly. One was withdrawn with persistent vomiting and diarrhoea, one with diabetes mellitus and one for a death unrelated to the study for which no post-mortem examination was allowed by the owner. One was withdrawn with poor owner compliance with drug administration.

\section{DISCUSSION}

The study reported herein showed a statistically significant increase in tear production during per os administration of cyclosporine at $5 \mathrm{mg} / \mathrm{kg}$ and a substantial improvement in ocular surface health in these dogs commensurate with their improvement in tear production. As the administration frequency of the drug was reduced, tear production fell, but still remained above baseline values with a similar alteration in the signs of corneal and conjunctival health over the study.

One might argue that the improvement in clinical signs was caused by the use of the ocular surface lubricant Viscotears (Allergan, New York). This was used to ensure some ocular surface lubrication even if the systemic cyclosporine administered had not resulted in increased tear production. This was essential for ethical reasons and yet, given its use, it is possible that it could have masked deleterious signs of tear film deficiency in the dogs. This is unlikely however, given that the lubricant was used three times daily throughout the study, and yet when the dose frequency of the cyclosporine was reduced, ocular signs of tear film deficiency such as ocular redness and increased ocular discharge were again noted.

It might be asked why one should investigate the ocular effects of cyclosporine given per os, since the drug, given topically twice daily, is highly effective in ameliorating the signs of canine KCS. Yet a number of owners find twice daily topical treatment difficult especially in dogs in which ocular surface pathology is painful and regular topical treatment is thus 
uncomfortable for the animal. In addition it might be hoped that systemic medication could markedly reduce the frequency of administration to a dosing rate considerably lower than twice daily, this being of benefit to the patient and owner. This was not found to be the case although the administration of cyclosporine per os once daily was considered substantially preferable by eight of the owners of dogs in the study, compared with the concept of twice daily topical administration. Two dogs were withdrawn from the study because of lack of drug efficacy but this was at the point of reducing from every other day to twice weekly treatment and on daily treatment they had shown excellent response to treatment.

Most of the dogs experienced some gastrointestinal side effects at the beginning of the study with slight vomiting and loose faeces, if not frank diarrhoea over a short time period, a recognised side-effect seen during the early period of cyclosporine therapy in dogs (Steffan et al.., 2006). Two dogs experienced more prolonged side effects and were withdrawn from the study and one died of causes unrelated to the study after an orthopaedic injury. One developed diabetes mellitus during the study which was considered coincidental. While human patients on per os cyclosporine for transplant rejection have previously been reported to develop diabetes mellitus (Perfornis and Kury-Paulin 2006), this is caused by insulin resistance and occurs at much higher levels of cyclosporine than that given in this study. The dog affected in this study was responsive to injectable insulin and thus the condition was considered extremely unlikely to have been caused by the drug.

Following the successful use of cyclosporine administered per os in this study, we have utilised this method of drug administration in four dogs with KCS where aggression made topical administration of any medication dangerous. In these animals oral cyclosporine has been beneficial as a sole agent. Clearly such anecdotal evidence requires further confirmation, but this, together with the findings of this study on the increase of tear production using the drug per os, suggests that oral cyclosporine alone is likely to be sufficient for the treatment of canine KCS.

\section{CONCLUSION}

In conclusion this study has demonstrated that cyclosporine given per os can have lacrimogenic effects resulting in beneficial effects for dogs with KCS. Further studies comparing the efficacy of the drug orally and topically are clearly warranted.

\section{REFERENCES}

[1] Berdoulay, A., English, R.V., Nadelstein, B. 2005. Effect of topical $0.02 \%$ tacrolimus aqueous suspension on tear production in dogs with keratoconjunctivitis sicca. Vet Ophthalmol. 8:225-32

[2] Gelatt, K.N., Peiffer, R.L. Jr., Erickson, J.L., Gum, G.G. 1975. Evaluation of tear formation in the dog, using a modification of the Schirmer tear test. J Am Vet Med Assoc 15;166:368-70

[3] Glen, J.B., Lawson, D.D. 1971. A modified technique of parotid duct transposition for the treatment of keratoconjunctivitis sicca in the dog. Vet Rec. 88:210-3.

[4] Hardie, R.J., Gregory, S.P., Tomlin, J., Sturgeon, C., Lipscomb, V., Ladlow, J. 2005.

[5] Cyclosporine treatment of anal furunculosis in 26 dogs. J Small Anim Pract. 46:3-9

[6] Hartley, C., Williams, D.L., Adams, V. 2006. Effect of age, gender, weight, and time of day on tear production in normal dogs. Vet Ophthalmol. 9:53-7.

[7] Kaswan, R.L., Martin, C.L., Chapman, W.L. Jr. 1984. Keratoconjunctivitis sicca: histopathologic study of nictitating membrane and lacrimal glands from 28 dogs. Am J Vet Res. 45:112-8

[8] Kaswan, R.L., Salibury, M-A., Ward, D. 1989. Spontaneous canine keratoconjunctivitis sicca. A useful model for human keratoconjunctivitis sicca: treatment with cyclosporine eye drops. Arch Ophthalmol. 107:1210-6

[9] Nell, B., Walde, I., Billich, A., Vit, P., Meingassner, J.G. 2005. The effect of topical pimecrolimus on keratoconjunctivitis sicca and chronic superficial keratitis in dogs: results from an exploratory study. Vet Ophthalmol. $8: 39-46$.

[10] Olivero, D.K., Davidson, M.G., English, R.V., Nasisse, M.P., Jamieson, V.E., Gerig, T.M. 1991. Clinical evaluation of $1 \%$ cyclosporine for topical treatment of keratoconjunctivitis sicca in dogs. J. Am. Vet. Med. Assoc. 199:1039-42.

[11] Olivry, T., Steffan, J., Fisch, R.D., Prélaud, P., Guaguère, E., Fontaine, J., Carlotti, D.N. 2002. European Veterinary Dermatology Cyclosporine Group Randomized controlled trial of the efficacy of cyclosporine in the treatment of atopic dermatitis in dogs. J Am Vet Med Assoc. 221:370-7.

[12] Penfornis, A., Kury-Paulin, S. 2006. Immunosuppressive drug-induced diabetes. Diabetes Metab. 32(5 Pt 2):539-46 
[13] Perry, H.D., Solomon, R., Donnenfeld, E.D., Perry, A.R., Wittpenn, J.R., Greenman, H.E., Savage, H.E. 2008. Evaluation of topical cyclosporine for the treatment of dry eye disease. Arch Ophthalmol. 126:1046-50.

[14] Radziejewski, K., Balicki, I. 2016 Comparative clinical evaluation of tacrolimus and cyclosporine eye drops for the treatment of canine keratoconjunctivitis sicca. Acta Vet Hung. 64:313-329

[15] Sansom, J., Barnett, K.C., Neumann, W., Schulte-Neumann, A., Clerc, B., Jegou, J.P., de Haas, V., Weingarten, A. 1995. Treatment of keratoconjunctivitis sicca in dogs with cyclosporine ophthalmic ointment: a European clinical field trial. Vet Rec. 137:504-7

[16] Salzberg, M., Muller, E. 2003. Quality requirements in clinical studies: a necessary burden? Swiss Med Wkly. 131:429-32

[17] Slatter, D., Severin, G.A. 1995. Use of pilocarpine for treatment of keratoconjunctivitis sicca. J Am Vet Med Assoc. 206:287-9.

[18] Steffan, J., Parks, C., Seewald, W. 2005. North American Veterinary Dermatology Cyclosporine Study Group Clinical trial evaluating the efficacy and safety of cyclosporine in dogs with atopic dermatitis. J Am Vet Med Assoc. 226:1855-63

[19] Steffan, J., Favrot, C., Mueller, R. 2006. A systematic review and meta-analysis of the efficacy and safety of cyclosporin for the treatment of atopic dermatitis in dogs. Vet Dermatol. 17:3-16.

[20] Termote, S. 2003. Parotid salivary duct mucocoele and sialolithiasis following parotid duct transposition. J Small Anim Pract. 44:213.

[21] Williams, D.L. 1997. A comparative approach to topical cyclosporine therapy. Eye. 11 ( $\mathrm{Pt}$ 4):453-64.

[22] Williams, D.L. 2008. Immunopathogenesis of keratoconjunctivitis sicca in the dog. Vet Clin N Am Small Anim Pract. 38:251-68

[23] Williams, D.L. and Tighe, A. (2018) Immuno histochemical evaluation of lymphocyte populations in the nictitans glands of normal dogs and dogs with keratoconjunctivitis sicca. Open Veterinary Journal In Press

[24] Williams, D.L. and Mann, B.K. (2014) Efficacy of a crosslinked hyaluronic acid-based hydrogel as a tear film supplement: a masked controlled study. PLoS One. 2014 Jun 10;9(6):e99766

[25] Williams, D., Middleton, S., Fattahian, H., Moridpour, R.(2012) Comparison of hyaluronic acid-containing topical eye drops with carbomer-based topical ocular gel as a tear replacement in canine keratoconjunctivitis sicca: A prospective study in twenty five dogs. Vet Res Forum. 3:229-32

Citation: David L Williams, Lacrimogenic Effects of Cyclosporine given per Os in Dogs with Keratoconjunctivitis Sicca. ARC Journal of Animal and Veterinary Sciences. 2017; 3(4):1-10. doi: dx.doi.org/ 10.20431/2455-2518.0304001.

Copyright: (c) 2017 Authors. This is an open-access article distributed under the terms of the Creative Commons Attribution License, which permits unrestricted use, distribution, and reproduction in any medium, provided the original author and source are credited. 